\title{
Implementing a reconfigurable Internet of Things Nodes using non-IP network based on Wireless Sensor Network
}

\author{
Khalida S. Rijab, Saif Muqdad Sadiq \\ Department of Electrical Engineering, University of Technology, \\ 30049@uotechnology.edu.iq., smuqdad7@gmail.com
}

\begin{abstract}
The idea of using wireless sensor network (WSN) let's as to upload multiple node's data over one IP, to reduce the number of needed IP multiple nodes, it's necessary to provide a backup sink for the data to let the sensors send their data via the link B when the link A is down. Therefore a web-portal has been designed with a debug terminal for the proposed IoT system to let us reconfigure the nodes and change the path of the wireless sensor network data. Two sink-nodes has been implemented for the proposed system, first one (A) for major data path that collected the data from sensors and uploads it to the web-portal and the second one (B) acts as a backup link for the data path. In the proposed system, we used the esp33 as a Wi-Fi connection for the wireless sensor network, and the NRF24 wireless transceiver module used to send the data from nodes to esp32.
\end{abstract}

Keywords: Internet of Things (IoT), Internet of Everything (IoE), wireless sensor network(WSN), reconfigurable embedded systems, web-portal.

Paper History:(Received :19-7-2018; Accepted: 2-102018)

\section{Introduction}

In recent years, one of the interesting research topics that also get an implication in key technological areas were the wireless sensor networks (WSN), its applications include telemedicine, automotive, environmental, domotics or military since their origin during the early 1980's into the Distributed Sensor Networks program at the Defense Advanced Research Projects Agency (DARPA) [1].

Wireless sensor networks (WSN) used in many applications for collecting data about the physical phenomena [2].

Also, the new generation of the modern communication systems (IoT) has a large development in recent years, there is a large number of applications for this attractive technology including the healthcare systems and environmental monitoring [3].

Their main goals include generating, transferring, collecting, analyzing and processing information from heterogeneous sensor nodes, using wireless networks to have signal processing, localization, or object and event tracking capabilities [4].
Normally, the WSN node architecture consists of an embedded processor, a communication device, and some interfaces, constrained to minimize the power consumption. However, the increased request for new applications that require more computational power and more adaptability of the node [5], this approach has been carried out in recent years.

Adding scalability and flexibility to the node architecture can increase the sensor node performance consequently, extensive work has been conducted recently to develop flexible designs [6].

There are real-time constraints on the embedded systems function procedure because of the advances in this network. [7].

A specified number of tasks as any real-time system executes the performed services. Real-time embedded tasks executed generally under time constraints represented by deadlines, limited memory, and batteries that provide low energy [8].

The design of the WSN keeps evolving because of the variation in their applications and the increase in their requirements, the concept of reconfiguration is considered as the recent and more effective evolution [9], the introduced concept of reconfigurations is related to the mode change theory [10]

In this theory, a system is included by the ability to switch between modes of execution. Within a specific mode, removing/adding tasks and/or changing their parameters are the basic procedures that take place [11]. However, the sudden changes required by the reconfiguration scenarios can severely alter the functions of the nodes.

In dynamic WSN, the self-adaptation and reconfiguration are vital capabilities of sensors by varying their functional and extra-functional (real-time and energy) properties [12].

Therefore, software architectural solutions are usually used to monitor and guide the dynamic changes to any WSN oriented application [13].

\section{Related Work}

In recent years, many papers published in this field like, Trong Nhan Le, 2016 [14] Published a work of a new 
Reconfigurable Directional Antenna based ReceiverInitiated Cycled Receiver (RDA-RICER) and used a low complexity and energy efficient scanning process in RDA-RICER to identify the direction providing the highest RSSI (Received Signal Strength Indicator) between two nodes.

Hanen Grichi with a research group, 2016 [15] explained a new algorithm that adjusts dynamically their actions to their environment under various conditions for the development of reconfigurable WSN. For that, they formulate a metamodel of RWSN based on UML to describe a zone-based architecture that uses a communication protocol for the optimization of distributed reconfiguration. they applied their concept to a case study, which is simulated with TRMSim-WSN and UPPAAL environment to expose the originality of this new architecture.

Also, G. Sridevi, 2018, [16] designed a reconfigurable interface with smart sensor for the industrial WSN in IoT systems. The system can collect the sensor data intelligently since the IEEE1451 protocol is embedded, the system implemented with CPLD and wireless communication applications. This can be used mostly in the real-time cases in the high-speed data acquisition system in IoT environments. With the water environmental application in the IoT environment, the system is thus verified and achieved the good effective results in the practical application.

As a research group 2018, [17], developed a metamorphosis of the internal architecture of the sensor nodes, also to any change the policy communication between the nodes aiming to adjust the network, this work improved the energy and real-time constraints.

In our proposed system, a reconfigurable WSN has been designed in an IoT application, the sensor nodes send their data to the sink node that forwards the data to the server to collect, process, and visualize the data in a webportal.

The reconfiguration operation in our proposed system could be performed by using the debug terminal that designed in the web-portal, by using specified commands, we can reconfigure the sink-nodes to let the data changes their sink path to save the data from the loss when the major link path was down.

\section{Proposed System}

Our proposed system consists of four sensor nodes, for application, the proposed system contains a two DHT12 (temperature and humidity sensor), DS18B20 (water temperature) and pzem-004t (AC Power Meter).

In addition, it contains two sink-nodes, first one for real data and the second acts as a backup link path for the data, figure (1) shows the block diagram of our proposed system.

The sensor nodes send their data to one of the two sinknodes by using the NRF24 modules, these modules use the SPI protocol that contains these pins, Master In Slave Out (MISO), Master Out Slave In (MOSI) and synchronous clock (SCK).

Any data that has been received by any one of the Sinknodes will be uploaded to the server using WIFI connection via the router
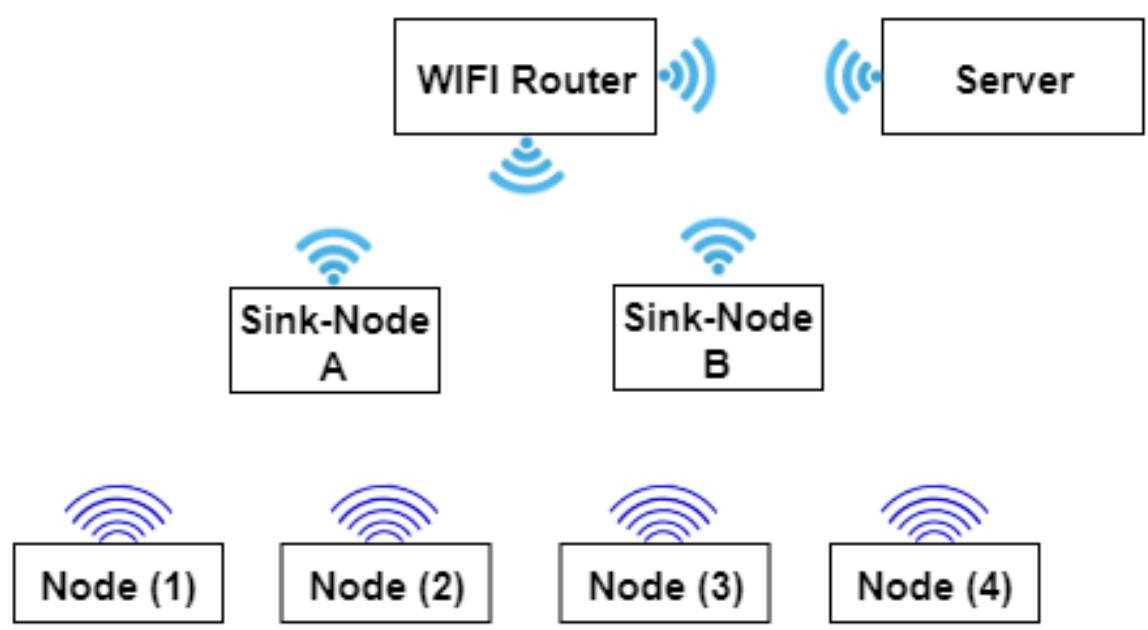

Figure (1): shows the block diagram of our proposed system

The server receives the data and collects it in SQL database and process these data to be ready to visualized in a web-portal, the web-portal was designed using (HTML, CSS, PHP, and JAVASCRIPT) programming languages.
In our proposed system a debug Terminal has been designed in the web-portal, by using this debug terminal we can send commands to the sink-nodes using WIFI connection via the router.

Each command that sends from the Terminal the Sinknodes will replay with the action that associated with this 
command, the replays will appear in the next line in the Terminal as shown in figure (2).

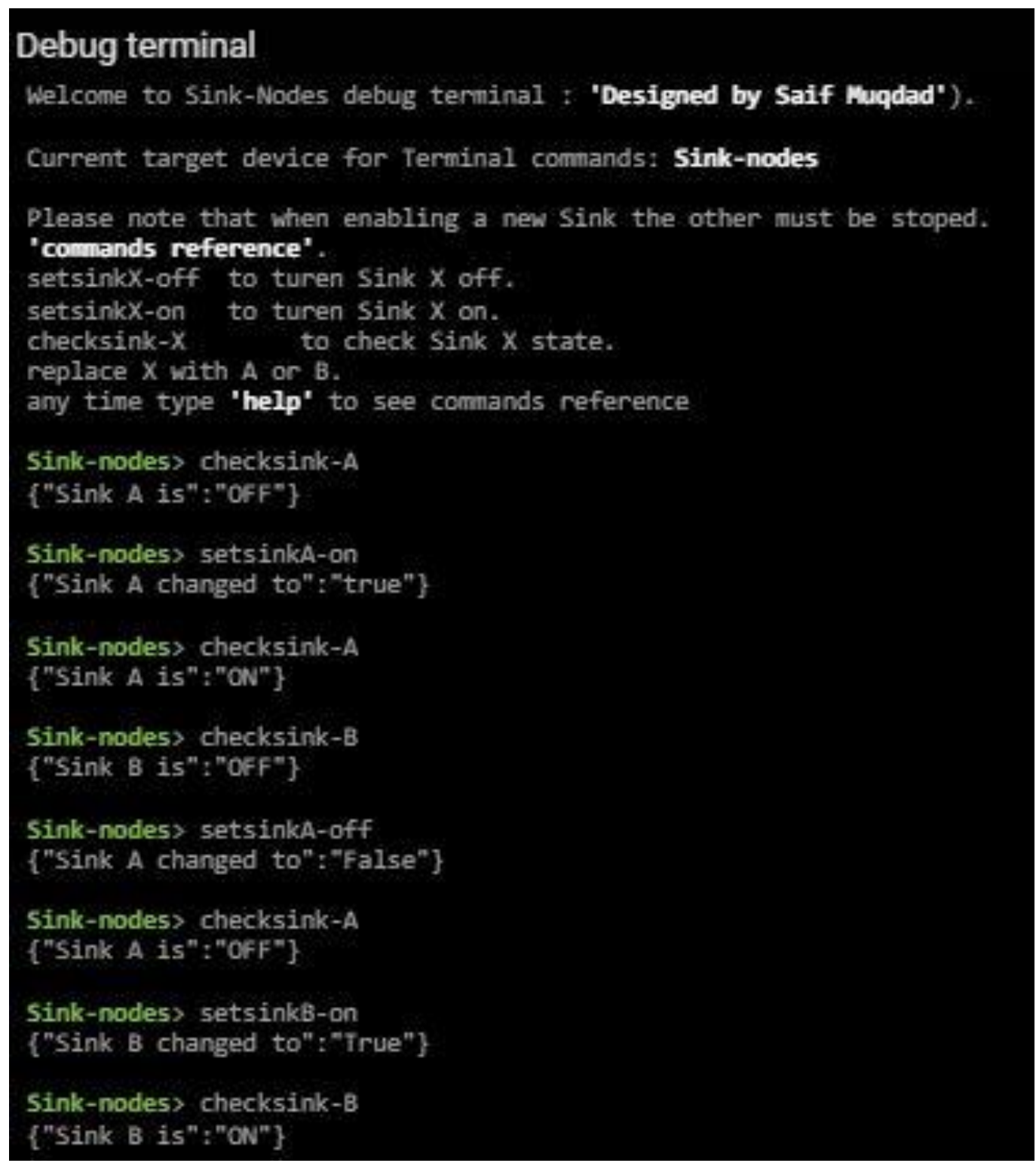

Figure (2): shows an example of sending a command and receive its associated replay

The configuration of the sink-nodes can be changed by using specified commands that have been defined in Sinknodes, by using this commands the user has the ability to open or close the path through any sink-node, also have the ability to check which nodes forwards the data now, figure (3): shows our proposed system flow chart. 


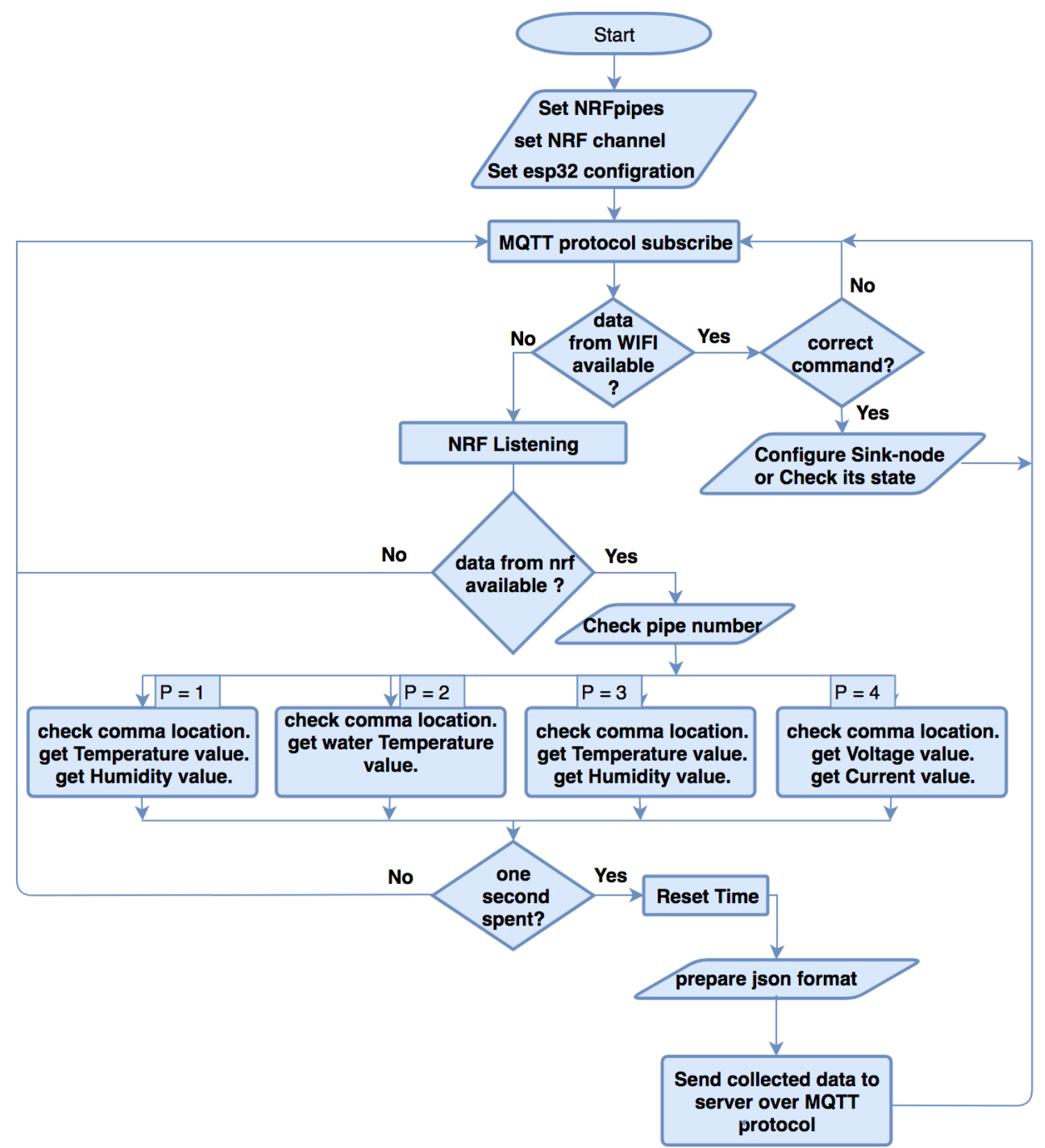

Figure (3): the flow chart of our proposed system

The proposed algorithm starts by setting the configuration parameters to the transceiver module, then the MQTT protocol let the Sink-node to be in the subscribe mode. The subscribe mode lets the Sink-node to be ready to receive data packets from the server. If there is a received data, it will be checked, if it is a correct commands the sink-node will execute the command and will replaying the server, but if it's not correct it will replays the server that there is error. If there is no packets coming from the server, the sink node will be ready to receive the packets of the data from the sensor nodes.

A reference of the commands that allowed by the sinknodes has been added to the debug terminal, any user all he needs to type "help" in debug terminal, the terminal will replays by the command reference as shown in figure (4). 


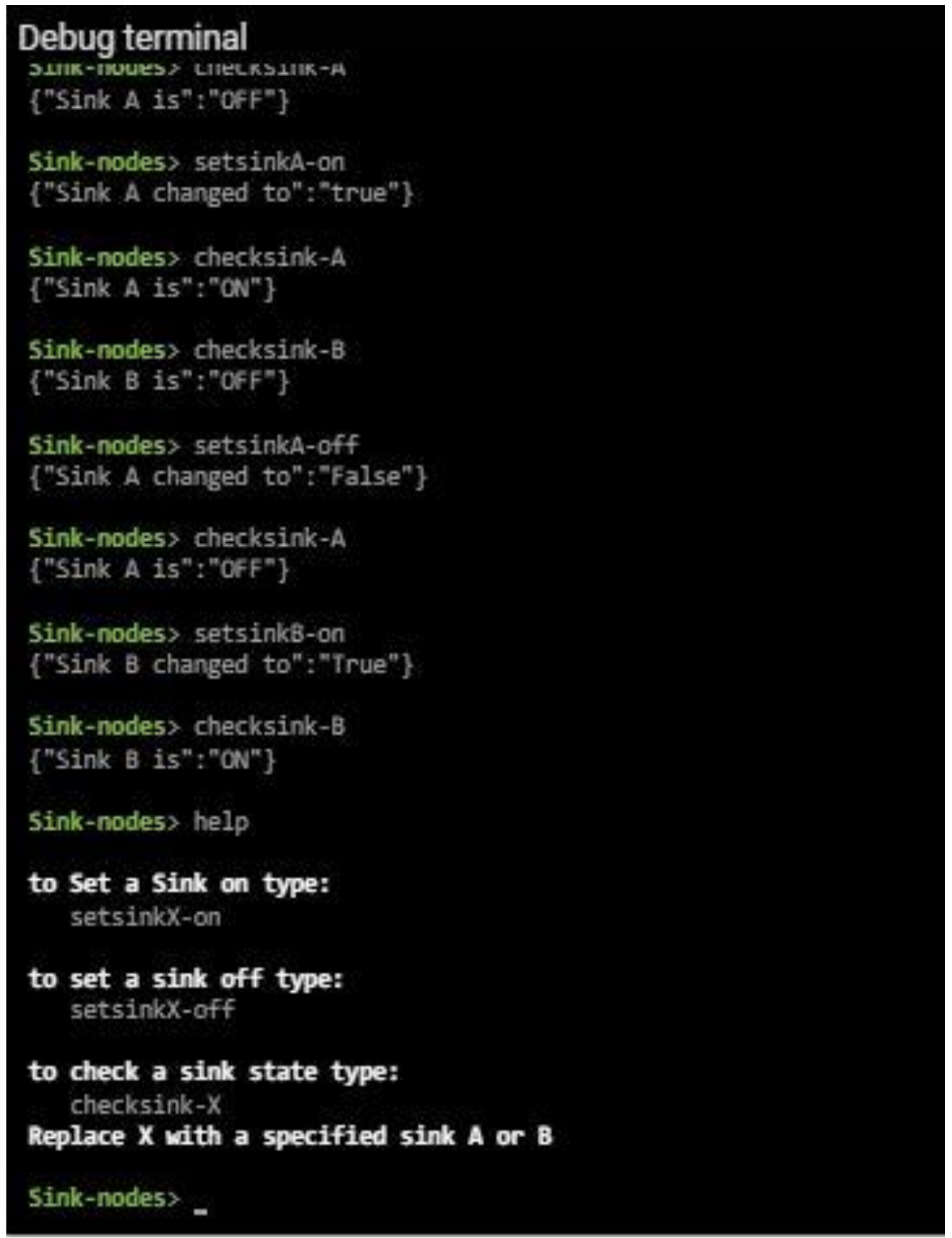

Figure (4): shows the reference commands of our proposed terminal

\section{Results}

By using our proposed wireless configuration system, the data rate that is required for the configuration has been reduced, this reduction can be explained as follows:-

The proposed system uses one sink-node that it is connected to the server over only IP address.

The Sink-node make the reconfiguration to change the path of four sensor nodes by using non-IP network.

The communication between the sink-node and the server need the source and destination MAC address and the source and the destination IP address. The communication between the Sink-node and the sensor nodes need only the pipe address, it is non-IP network.

The size of the one MAC address is 6 byte, there are two MAC address in the packet between the server and the sink node, therefore the communication uses 12 byte only for MAC address. The size of the IP address is 4 bytes, then the system transmitting 8 bytes only for IP address. This means for each communication between two IP network nodes, there are 20 bytes only for addressing, if there is 4 nodes the system will use 80 bytes only for addressing, this bytes as we is transmitted every second. 
In our proposed system, the system uses the IP network only for the sink-node and then the sink node will do the configuration to change the path of four sensor nodes by using non-IP address network. The modules that are used in the proposed system to make the communication between the sink node and the sensor node are the NRF24 transceiver modules, it uses just 5 bytes for addressing, then the transmitted bits per seconds will be reduced in our proposed design against the general design that is depends only one the IP network.

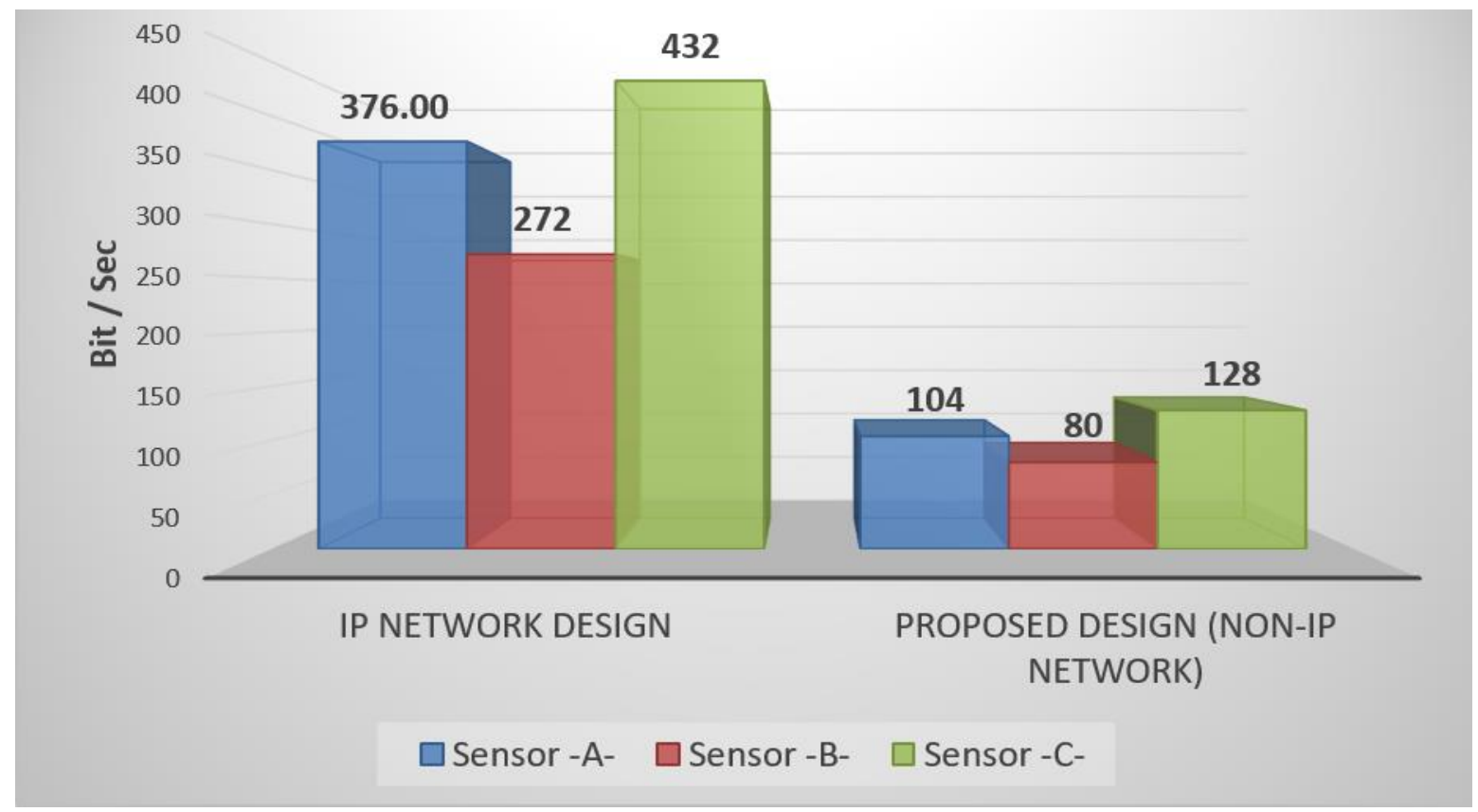

Figure (5): shows the comparison of transmitted bit per second between the IP network designs with the proposed design.

-A- temperature and Humidity sensor node.

-B- Water temperature sensor node.

-C- Voltage and Current sensor node.

In addition, the IP network uses the JSON format for transmitting the information while the non-IP network transmitting the data directly. Then the transmitted bits will be also reduced as a result for the number of bits that it is in the data packet that uses the JSON format, the figure (4) explains that the proposed system reduces the data transmission rate against the general design that are explained in the literature review. When the reduction has been calculated for the general and proposed design, it appears that the proposed system reduces the data rate nearly up to $70 \%$ against the general design.

\section{Discussion}

Our proposed system combines the concept of the reconfigurable Wireless Sensor Network (RWSN) with Internet Of Things (IoT) applications, Also it has been explained that it has the ability to be reconfigured wirelessly by using the debug terminal that designed in web-portal.

Our proposed system shows that it's easy to change the path of the sink data by using a simple command, also our designed terminal increase the ease of using the commands by typing the command reference to the user when user type "help" in the terminal.

Our proposed IoT system looks like a tree WSN architecture, it's necessary to provide a backup sink path for cases when a maintains has been needed to the sinknode, also we surely need a backup path link for cases when the major path was down to prevent the loss of the data.

\section{Conclusion and future work}

In this paper, we proposed a design of a portable and lowcost IoT application, and we prototyped a sensor node.

A Web-portal has been designed to our IOT application and it includes a debug terminal that lets us reconfigure the sink-nodes, We used multiple sensor nodes each node contain one sensor, a temperature and humidity sensor, a water temperature sensor, and $\mathrm{AC}$ power meter sensor. 
We suggest in future work to add the ability to the system to make a load balance by letting some of the sensor nodes send data to the Sink -A- and other sensor nodes sends there data through sink -B-.

In addition, it is very exciting by adding some intelligent by letting the system itself doing the load balance according to the amount of data that comes from each sensor node.

\section{References}

1. A. Kumar, H, Kim, \& P. Hancke, "Environmental Monitoring Systems: A Review," in IEEE Sensors Journal, Vol. 13(4), 2013, pp. 1329-1339.

2. Wang. L., Xu. L. D., Bi. Z., Xu. Y.: "Data cleaning for RFID and WSN integration”. IEEE Trans. Ind. Informat., vol. 10 , no. 1,2014 , pp. 408-418.

3. He. W., Yan. G., Xu. L.: "Developing vehicular data cloud services in the IoT environment", IEEE Trans. Ind. Informat., vol. 10, no. 2,2014, pp. 1587-1595.

4. P. Bellavista, \& G. Cardone, "Convergence of MANET and WSN in IoT Urban Scenarios," in Sensors Journal, Vol. 13(10),2013, pp. 3558-3567.

5. I.F. Akyildiz, T. Melodia, \& K.R. Chowdhury, "A survey on wireless multimedia sensor networks," in Computer Networks, Vol. 51(4), 2007, pp. 921960 .

6. Andreas Brokalakis, Georgios-Grigorios Mplemenos, Konstantinos Papadopoulos and Ioannis Papaefstathiou, "RESPONSE: An Innovative, Reconfigurable, Powerful and Energy Efficient WSN Node", in IEEE, 2011.

7. D. J. Hatley and I. A. Pirbhai, "Strategies for RealTime System Specification", New York, NY, USA: Addison-Wesley, 2013.

8. L. He et al., "ESync: An energy synchronized charging protocol for rechargeable wireless sensor networks," in Proc. ACM Int. Symp. Mobile Ad Hoc Netw. Comput., Philadelphia,2014.

9. M. Chen, "Reconfiguration of sustainable thermoelectric generation using wireless sensor network," IEEE Trans. Ind. Electron., vol. 61, no. 6, pp. 2776-2783.

10. L. Pezzarossa, M. Schoeberl, and J. Sparso, "Towards utilizing reconfigurable shared resources in multi-core hard real-time systems," in Proc. Junior Researcher Workshop Real-Time Comput., Lille, France, 2015.

11. S. B. Meskina, N. Doggaz, M. Khalgui, and Z. Li, "Multiagent framework for smart grids recovery,"
IEEE Trans. Syst., Man, Cybern., Syst. vol. 47, no. 7, 2017, pp. 1284-1300, Jul.

12. W. Housseyni, O. Mosbahi, M. Khalgui, Z. Li, and L. Yin, "Multiagent architecture for distributed adaptive scheduling of reconfigurable real-time tasks with energy harvesting constraints," IEEE Access, vol. 6, 2017, pp. 2068-2084.

13. I. Ghribi et al., "R-codesign: Codesign methodology for real-time reconfigurable embedded systems under energy constraints," IEEE Access, vol. 6, 2018, pp. 14078-14092.

14. Trong Nhan Le, Alain Pegatoquet, Trinh Le Huy, Leonardo Lizzi and Fabien Ferrero, "Improving Energy Efficiency of Mobile WSN Using Reconfigurable Directional Antennas", IEEE communication letter, JUNE 2016.

15. Hanen Grichi, Olfa Mosbahi, Mohamed Khalgui, and Zhiwu Li, Fellow, IEEE, "RWiN: New Methodology for the Development of Reconfigurable WSN", 2016.

16. G. Sridevi, S. Satyanarayana, and P. Sravan Kumar, "Implementing the Reconfigurable Intelligent Sensor Interface in Wireless Networks", 2018.

17. Maroua Gasmi, Olfa Mosbahi, Mohamed Khalgui, Luis Gomes, Senior Member, IEEE, and Zhiwu Li, Fellow, IEEE, "Performance Optimization of Reconfigurable Real-Time Wireless Sensor Networks", in IEEE 2018. 